\title{
On the Concept of a Notational Variant
}

Alex Kocurek For the 6th International Workshop in Logic, Rationality, and Interaction.

Abstract. In the study of modal and nonclassical logics, translations have frequently been employed as a way of measuring the inferential capabilities of a logic. It is sometimes claimed that two logics are "notational variants" if they are translationally equivalent. However, we will show that this cannot be quite right, since first-order logic and propositional logic are translationally equivalent. Others have claimed that for two logics to be notational variants, they must at least be compositionally intertranslatable. The definition of compositionality these accounts use, however, is too strong, as the standard translation from modal logic to first-order logic is not compositional in this sense. In light of this, we will explore a weaker version of this notion that we will call schematicity and show that there is no schematic translation either from first-order logic to propositional logic or from intuitionistic logic to classical logic.

\section{$\S 1$ Introduction}

In the study of modal and nonclassical logics, translations (maps between formulas that faithfully preserve consequence) are frequently employed as a way of measuring the inferential capabilities of a logic. Examples of well-known translations in the literature include:

(a) the double-negation translation of classical logic into intuitionistic logic;

(b) the standard translation of modal logic into first-order logic;

(c) the Gödel translation of intuitionistic logic into classical S4.

These translations are often taken to show that the logic being translated can be viewed as a "notational variant" of a fragment of the logic it is translated into. Indeed, a number of authors have conjectured that translational equivalence is a necessary and/or sufficient condition for two logics to be notational variants in the intuitive sense. ${ }^{1}$

Unfortunately, most of these accounts of notational variance are either too weak or too strong. For instance, on any reasonable theory of notational variance, first-order logic and propositional logic are not notational variants. However, we will show in $\S 3$ that first-order logic and propositional logic are translationally equivalent. Thus, any account which says translational equivalence is sufficient for notational variance ${ }^{2}$ is too weak.

On the other hand, some have suggested that for two logics to be considered notational variants, they must at least be compositionally intertranslatable, in a sense that will be made

\footnotetext{
* Thanks to Wes Holliday and two anonymous reviewers for their helpful feedback on this paper.

${ }^{1}$ For claims like this, see Wójcicki [1988, p. 67], Epstein [1990, p. 391], Pelletier and Urquhart [2003, p. 269], Caleiro and Gonçalves [2007, p. 108], Straßburger [2007, p. 139], Mossakowski et al. [2009, p. 7], and French [2010, p. 134].

2E.g., Straßburger [2007, p. 139], Mossakowski et al. [2009, p. 7], and French [2010, p. 134].
} 
precise in $\S 4 .{ }^{3}$ Since there is no compositional translation from first-order logic to propositional logic, the former is not a notational variant of the latter in this sense. However, these accounts of notational variance are too strong, since on their definition of compositionality, even the standard translation (in fact, any translation) of modal logic into first-order logic is not compositional. Near the end of this paper, a generalization of this notion called schematicity that avoids these problems will be proposed, and we will show that there is no schematic translation from first-order logic to propositional logic, or from intuitionistic logic to classical logic.

\section{§2 Defining Translations}

We start by defining the concept of a logic and a translation in abstract terms.

Definition 2.1 (Logic). A logic is a pair $\mathbf{L}=\langle\mathcal{L}, \models\rangle$ where $\mathcal{L}$ is a nonempty class (of formulas) and $\models \subseteq \wp(\mathcal{L}) \times \mathcal{L}$ (the consequence relation) such that:

(i) $\models$ is reflexive, i.e., for all $\phi \in \mathcal{L}, \phi \models \phi$

(ii) $\models$ is transitive, i.e., for all $\Gamma, \Delta \subseteq \mathcal{L}$ and all $\phi \in \mathcal{L}$, if $\Gamma \models \phi$ and if $\Delta \models \gamma$ for each $\gamma \in \Gamma$, then $\Delta \models \phi$.

Where $\phi, \psi \in \mathcal{L}$, we will say $\phi$ and $\psi$ are L-equivalent, written " $\phi \equiv \psi$ ", if $\phi \models \psi$ and $\psi \models \phi$. We will say that $\phi$ is $\mathbf{L}$-valid, written " $\models \phi^{\prime}$, if $\varnothing \models \phi$. If $\mathbf{L}$ is a logic, we may write " $\models_{\mathbf{L}}$ " and " $\equiv_{\mathbf{L}}$ " for the consequence and equivalence relations for $\mathbf{L}$ respectively. We may also write " $\models_{i}$ " instead of " $\models_{\mathbf{L}_{i}}{ }^{\prime}, " \equiv_{i}$ " instead of " $\equiv_{\mathbf{L}_{i}}$, , etc.

This notion of a logic is meant to be fairly general. While it can be generalized even further (allowing for substructural logics, multiple-conclusion logics, etc.), such generalizations will not concern us here. Classical, intuitionistic, modal, and predicate logics can all be viewed as logics in the sense of Definition 2.1.

Next, we define the concept of a translation.

Definition 2.2 (Translation). Let $\mathbf{L}_{1}$ and $\mathbf{L}_{2}$ be logics. A translation from $\mathbf{L}_{1}$ to $\mathbf{L}_{2}$ is a map t: $\mathcal{L}_{1} \rightarrow \mathcal{L}_{2}$ such that for all $\Gamma \subseteq \mathcal{L}_{1}$ and $\phi \in \mathcal{L}_{1}, \Gamma \models_{1} \phi$ iff $\mathrm{t}[\Gamma] \models_{2} \mathrm{t}(\phi)$. If $\mathrm{t}$ is a translation from $\mathbf{L}_{1}$ to $\mathbf{L}_{2}$, we will write " $t: \mathbf{L}_{1} \rightsquigarrow \mathbf{L}_{2}$ ". We will say $\mathbf{L}_{1}$ is translatable into $\mathbf{L}_{2}$, written as " $\mathbf{L}_{1} \rightsquigarrow \mathbf{L}_{2}$ ", if there is a translation from $\mathbf{L}_{1}$ to $\mathbf{L}_{2}$. We will say $\mathbf{L}_{1}$ and $\mathbf{L}_{2}$ are intertranslatable, written as " $\mathbf{L}_{1} \stackrel{\rightsquigarrow}{\rightsquigarrow} \mathbf{L}_{2}$ ", if $\mathbf{L}_{1} \rightsquigarrow \mathbf{L}_{2}$ and $\mathbf{L}_{2} \rightsquigarrow \mathbf{L}_{1}$.

Example 2.3 (Double-Negation Translation). Define At $=\left\{p_{0}, p_{1}, p_{2}, \ldots\right\}$. Let $\mathcal{L}_{\text {Prop }}$ be the set of formulas defined recursively over At as follows:

$$
\phi::=p|\neg \phi|(\phi \wedge \phi) \text {. }
$$

\footnotetext{
${ }^{3}$ E.g., Wójcicki [1988, p. 67], Epstein [1990, p. 391], Pelletier and Urquhart [2003, p. 269], and Caleiro and Gonçalves [2007, p. 108].
} 
Let CPL be classical propositional logic over $\mathcal{L}_{\text {Prop }}$, and let IPL be intuitionistic propositional logic over $\mathcal{L}_{\text {Prop }}(\vee, \rightarrow)$, i.e., the result of extending $\mathcal{L}_{\text {Prop }}$ with connectives $\vee$ and $\rightarrow$. Define $\mathrm{t}(\phi):=\neg \neg \phi$. Then $\mathrm{t}: \mathrm{CPL} \rightsquigarrow$ IPL.

Example 2.4 (Standard Translation). Let Var $=\left\{x_{0}, x_{1}, x_{2}, \ldots\right\}$ (the set of variables) and for each $n \in \mathbb{N}$, let $\operatorname{Pred}^{n}=\left\{P_{0}^{n}, P_{1}^{n}, P_{2}^{n}, \ldots\right\}$ (the set of $n$-place predicates). Define $\mathcal{L}_{\text {Pred }}$ to be the set of formulas defined recursively as follows:

$$
\phi::=P^{n}\left(y_{1}, \ldots, y_{n}\right)|\neg \phi|(\phi \wedge \phi) \mid \forall x \phi .
$$

Let FOL be classical first-order logic over $\mathcal{L}_{\text {Pred }}$. Define $\mathcal{L}_{\text {Prop }}(\square)$ to be the set of formulas defined recursively over At as follows:

$$
\phi::=p|\neg \phi|(\phi \wedge \phi) \mid \square \phi .
$$

Let $\mathrm{K}$ be the minimal normal modal logic over $\mathcal{L}_{\text {Prop }}(\square)$. Where $R$ is an arbitrarily chosen binary predicate and where $n \in \mathbb{N}$, we define the map $S T_{n}$ from propositional modal formulas to first-order formulas as follows:

$$
\begin{aligned}
S T_{n}\left(p_{i}\right) & =P_{i}^{1}\left(x_{n}\right) \\
S T_{n}(\neg \phi) & =\neg S T_{n}(\phi) \\
S T_{n}(\phi \wedge \psi) & =\left(S T_{n}(\phi) \wedge S T_{n}(\psi)\right) \\
S T_{n}(\square \phi) & =\forall x_{n+1}\left(R\left(x_{n}, x_{n+1}\right) \rightarrow S T_{n+1}(\phi)\right) .
\end{aligned}
$$

Then $S T_{n}: \mathbf{K} \rightsquigarrow$ FOL.

Example 2.5 (Non-normal Modal Logics). A modal logic over $\mathcal{L}_{\text {Prop }}(\square)$ is said to be monotonic if it contains all classical tautologies as well as the axiom $\square(p \wedge q) \rightarrow$ $(\square p \wedge \square q$ ) and it is closed under uniform substitution, modus ponens, and the rule $\phi \leftrightarrow \psi / \square \phi \leftrightarrow \square \psi$. Kracht and Wolter Kracht and Wolter [1999, p. 109, Theorem 4.7] showed that the following map is a translation from any monotonic modal logic to a normal bimodal logic (i.e., a modal logic over the language $\mathcal{L}_{\text {Prop }}\left(\square_{1}, \square_{2}\right)$, where each $\square_{i}$ is a normal modal operator):

$$
\begin{aligned}
\mathrm{t}(p) & =p \\
\mathrm{t}(\neg \phi) & =\neg \mathrm{t}(\phi) \\
\mathrm{t}(\phi \wedge \psi) & =\mathrm{t}(\phi) \wedge \mathrm{t}(\psi) \\
\mathrm{t}(\square \phi) & =\diamond_{1} \square_{2} \mathrm{t}(\phi) .
\end{aligned}
$$

Thomason Thomason $[1974,1975]$ also shows how to translate any tense logic into a normal (mono)modal logic, though the translation is too complex to state succinctly here. 
Logicians have typically taken the existence of such translations to show that the source logic is a mere notational variant of a fragment of the target logic. Gödel [1933] (reprinted in Gödel [1986]) says of the translation from CPL to IPL:

If to the primitive notions of Heyting's propositional calculus we let correspond those notions of the classical propositional calculus that are denoted by the same sign and if to absurdity $(\neg)$ we let correspond negation $(\sim)$, then the intuitionistic propositional calculus $H$ turns out to be a proper subsystem of the ordinary propositional calculus $A$. With another correlation (translation) of the notions, however, the classical propositional calculus is, conversely, a subsystem of the intuitionistic one. Gödel [1986, p. 287]

Blackburn et al. [2001, p. xi] say of the standard translation from modal logic to first-order logic:

By adopting the perspective of correspondence theory, modal logic can be regarded as a fragment of first- or second-order classical logic.

Kracht and Wolter [1999, p. 100] informally explain the significance of their result that monotonic modal logics are translatable into normal bimodal logics as follows:

The positive results on simulations [i.e., translations] show that there is no essential difference between the classes of monomodal normal logics, monotonic logics, and polymodal logics.

Finally, Thomason [1975, p. 154] summarizes his result that tense logics are translatable into normal (mono)modal logics as follows:

In general terms, these results would seem to indicate that there is nothing to be gained by considering many modalities rather than just one, except simplicityanything which can be expressed about the universe in terms of many notions of necessity can be expressed in terms of one, very complex, notion of necessity, by a translation which preserves both the semantic and syntactic consequence relations.

Although we will show that translations between logics are fairly easy to come by, there are non-trivial failures of translatability. For instance, Jeřábek [2012, p. 672] showed that there is no translation from CPL to the logic of paradox LP. As another example, the following is readily verified:

Proposition 2.6. If $\mathbf{L}_{1} \rightsquigarrow \mathbf{L}_{2}$, then $\mathbf{L}_{2}$ is compact only if $\mathbf{L}_{1}$ is.

From this, it follows that second-order logic is not translatable into first-order logic. Moreover, as the next example shows, there are pairs of logics such that neither logic is translatable into the other. ${ }^{4}$

\footnotetext{
${ }^{4}$ This thereby answers a question posed by Epstein [1990, p. 388] in the affirmative. It is also straightforward to generate artificial counterexamples using any two partial orders such that neither is order-embeddable in the other.
} 
Example 2.7 (Kleene Logic). Let $\mathrm{K} 3$ be the strong Kleene logic over $\mathcal{L}_{\text {Prop }}$. Let us write $\mathbf{C P L}^{n}$ and $\mathbf{K} 3^{n}$ for the logics obtained from $\mathbf{C P L}$ and $\mathbf{K} 3$ respectively by restricting the set of formulas to those whose atomics are all among $\left\{p_{1}, \ldots, p_{n}\right\}$. Then neither $\mathbf{C P L}{ }^{n}$ nor $\mathbf{K} 3^{n}$ is translatable into the other. $\mathbf{C P L}{ }^{n}$ is not translatable into $\mathbf{K} \mathbf{3}^{n}$ since there are no tautologies in $\mathbf{K} 3^{n}$ (this generalizes to CPL and K3). And $\mathbf{K} 3^{n}$ is not translatable into $\mathrm{CPL}^{n}$ since the former has strictly more formulas up to equivalence than the latter (this does not generalize to CPL and K3; in fact, K3 $\rightsquigarrow$ CPL by Theorem 3.5 below).

One might conjecture that two logics are notational variants if they are intertranslatable. However, a number of authors have claimed that intertranslatability is not enough for two logics to be properly called "notational variants". Rather, they must additionally be translationally equivalent in the following sense: ${ }^{5}$

Definition 2.8 (Translational Equivalence). Let $\mathbf{L}_{1}$ and $\mathbf{L}_{2}$ be logics. We will say that $\left\langle t_{1}, t_{2}\right\rangle$ is a translation scheme between $L_{1}$ and $L_{2}$ (written as " $t_{1}, t_{2}$ : $L_{1}$ « $\mathrm{t}_{1}: \mathbf{L}_{1} \rightsquigarrow \mathbf{L}_{2}$ and $\mathrm{t}_{2}: \mathbf{L}_{2} \rightsquigarrow \mathbf{L}_{1}$ and for all $\phi \in \mathcal{L}_{1}$ and all $\psi \in \mathcal{L}_{2}$ :

$$
\begin{aligned}
& \mathrm{t}_{2}\left(\mathrm{t}_{1}(\phi)\right) \equiv_{1} \phi \\
& \mathrm{t}_{1}\left(\mathrm{t}_{2}(\psi)\right) \equiv_{2} \psi .
\end{aligned}
$$

$\mathbf{L}_{1}$ and $\mathbf{L}_{2}$ are translationally equivalent (written " $\mathbf{L}_{1} \leadsto \mathbf{L}_{2}$ ") if $t_{1}, t_{2}: \mathbf{L}_{1} \leadsto \mathbf{L}_{2}$ for some $t_{1}$ and $t_{2}$.

Translational equivalence is strictly stronger than intertranslatability. In particular, as we will now show, CPL and IPL are intertranslatable but not translationally equivalent.

Definition 2.9 (Lindenbaum-Tarski Algebra). Let $\mathbf{L}=\langle\mathcal{L}, \models\rangle$ be a logic. The LindenbaumTarski algebra of $\mathbf{L}$ is the poset $\mathbb{L}_{\mathbf{L}}=\langle\mathcal{L} / \equiv, \leqslant\rangle$ where $\mathcal{L} / \equiv$ is the class of $\equiv$-classes on $\mathcal{L}$ and where $[\phi]_{\mathrm{L}},[\psi]_{\mathrm{L}} \in \mathcal{L} / \equiv,[\phi]_{\mathrm{L}} \leqslant[\psi]_{\mathrm{L}}$ iff $\phi \models \psi$ (it is easy to verify this is well-defined since $\models$ is transitive).

Proposition 2.10. CPL $\stackrel{\leftrightarrow}{\rightsquigarrow}$ IPL but not CPL $\rightsquigarrow$ IPL.

Proof: We saw above that CPL $\rightsquigarrow$ IPL via the double-negation translation. Moreover, by Theorem 3.5 below, IPL $\rightsquigarrow$ CPL. Thus, CPL $\stackrel{\rightsquigarrow}{\rightsquigarrow}$ IPL. Suppose $t, s:$ CPL $\rightsquigarrow$ IPL. Define $f: \mathbb{L}_{\mathrm{CPL}} \rightarrow \mathbb{L}_{\mathrm{IPL}}$ and $g: \mathbb{L}_{\mathrm{IPL}} \rightarrow \mathbb{L}_{\mathrm{CPL}}$ such that $f\left([\phi]_{\mathrm{CPL}}\right)=[\mathrm{t}(\phi)]_{\mathrm{IPL}}$ and $g\left([\phi]_{\mathrm{IPL}}\right)=[\mathrm{s}(\phi)]_{\mathrm{CPL}}$ (this is well-defined since translations preserve equivalence).

${ }^{5}$ See, e.g., Pelletier and Urquhart [2003, p. 269], Caleiro and Gonçalves [2007, p. 108], Straßburger [2007, p. 139], and French [2010, p. 134]. 
It is easy to check that $f$ and $g$ are order-embeddings such that $f\left(g\left([\phi]_{\text {IPL }}\right)\right)=[\phi]_{\text {IPL }}$ and $g\left(f\left([\phi]_{\mathbf{C P L}}\right)\right)=[\phi]_{\mathbf{C P L}}$. Thus, if $\mathbf{C P L} \leadsto \mathbf{I P L}$, then $\mathbb{L}_{\mathbf{C P L}}$ and $\mathbb{L}_{\mathbf{I P L}}$ would be order-isomorphic, $\{$.

There is an even stronger notion of equivalence between logics, viz., that of isomorphism:

Definition 2.11 (Isomorphism). We will say $\mathbf{L}_{1}$ is isomorphic to $\mathbf{L}_{2}$, written as " $\mathbf{L}_{1} \cong$ $\mathbf{L}_{2}{ }^{\prime \prime}$, if there is a bijective $t: \mathbf{L}_{1} \rightsquigarrow \mathbf{L}_{2}$.

Observe that if $\mathrm{t}: \mathbf{L}_{1} \rightsquigarrow \mathbf{L}_{2}$ is bijective, then $\mathrm{t}^{-1}: \mathbf{L}_{2} \rightsquigarrow \mathbf{L}_{1}$, and therefore $\mathrm{t}, \mathrm{t}^{-1}: \mathbf{L}_{1} \rightsquigarrow \mathbf{L}_{2}$. Thus, isomorphism implies translational equivalence. The converse can fail for trivial cardinality reasons. For example, let $\mathbf{C P L}^{*}$ be the result of adding uncountably many "redundant" unary operators $\triangle_{r}$ for each $r \in \mathbb{R}$ such that $\triangle_{r} \phi \equiv \equiv_{\mathrm{CPL}^{*}} \phi$. Then CPL $\leadsto \mathbf{C P L}^{*}$ but $\mathbf{C P L} \nRightarrow \mathbf{C P L}^{*}$. Yet intuitively, $\mathbf{C P L}$ is a notational variant of CPL. After all, each $\triangle_{r}$ is quite straightforwardly definable in CPL, and intuitively, adding definable operators to a logic does not yield a new logic. Hence, requiring notational variants to be isomorphic would be unreasonably restrictive. One would prefer a weaker notion of notational variance (such as translational equivalence) on which such artificial cardinality considerations are not deemed essential to a logic.

So suppose we stipulate for a moment that two logics are notational variants just in case they are translationally equivalent. We will now show that $\mathbf{L}_{1} \rightsquigarrow \mathbf{L}_{2}$ just in case $\mathbf{L}_{1}$ is a notational variant of a fragment of $\mathbf{L}_{2}$.

Definition 2.12 (Fragment). Let $\mathbf{L}_{1}$ and $\mathbf{L}_{2}$ be logics. We will say $\mathbf{L}_{1}$ is a fragment of $\mathbf{L}_{2}$ (written as " $\mathbf{L}_{1} \subseteq \mathbf{L}_{2}$ ") if (a) $\mathcal{L}_{1} \subseteq \mathcal{L}_{2}$, and (b) for all $\Gamma \subseteq \mathcal{L}_{1}$ and $\phi \in \mathcal{L}_{1}: \Gamma \models_{1} \phi$ iff $\Gamma \models_{2} \phi$.

Proposition 2.13. Let $\mathbf{L}_{1}$ and $\mathbf{L}_{2}$ be logics. Then the following are equivalent:
(a) $\mathbf{L}_{1} \rightsquigarrow \mathbf{L}_{2}$.
(b) There is an $\mathbf{L}_{2}^{\prime} \subseteq \mathbf{L}_{2}$ such that $\mathbf{L}_{1} \stackrel{\leftrightarrow}{\rightsquigarrow} \mathbf{L}_{2}^{\prime}$.
(c) There is an $\mathbf{L}_{2}^{\prime} \subseteq \mathbf{L}_{2}$ such that $\mathbf{L}_{1} \leadsto \mathbf{L}_{2}^{\prime}$.

Proof: Obviously, (c) implies (b), which implies (a) (since the composition of two translations is also a translation). To show that (a) implies (c), let $t: \mathbf{L}_{1} \rightsquigarrow \mathbf{L}_{2}$. Define $\mathbf{L}_{\mathrm{t}[1]}=\left\langle\mathrm{t}\left[\mathcal{L}_{1}\right], \models_{\mathrm{t}[1]}\right\rangle$ where $\mathrm{t}[\Gamma] \models_{\mathrm{t}[1]} \mathrm{t}(\phi)$ iff $\mathrm{t}[\Gamma] \models_{2} \mathrm{t}(\phi)$. By definition, $\mathbf{L}_{\mathrm{t}[1]} \subseteq$ $\mathbf{L}_{2}$. Hence, it suffices to show that $\mathbf{L}_{1} \leadsto \mathbf{L}_{\mathrm{t}[1]}$.

Now, $\mathrm{t}^{-1}$ (the inverse of $\mathrm{t}$ ) may not be a function from $\mathrm{t}\left[\mathcal{L}_{1}\right]$ to $\mathcal{L}_{1}$, since $\mathrm{t}$ might not be injective. But since $t^{-1}$ is total on $t\left[\mathcal{L}_{1}\right]$, we can always find a function $t^{*} \subseteq$ 
$\mathrm{t}^{-1}$ (using the axiom of choice) by selecting a $\psi \in\left\{\psi^{\prime} \in \mathcal{L}_{1} \mid \mathrm{t}\left(\psi^{\prime}\right)=\phi\right\}$ arbitrarily for each $\phi \in \mathbf{L}_{\mathrm{t}[1]}$ and setting $\mathrm{t}^{*}(\phi)=\psi$. Observe that $\mathrm{t}^{*}$ is a right-inverse of $\mathrm{t}$, i.e., for all $\phi \in \mathrm{t}\left[\mathcal{L}_{1}\right], \mathrm{t}\left(\mathrm{t}^{*}(\phi)\right)=\phi$. Using this fact, it is straightforward to verify that $\mathrm{t}, \mathrm{t}^{*}: \mathbf{L}_{1} \leadsto \mathbf{L}_{\mathrm{t}[1]}$.

Hence, if notational variance is translational equivalence, then to show that $\mathbf{L}_{1}$ is a translatable into $\mathbf{L}_{2}$ just is to show that $\mathbf{L}_{1}$ is a notational variant of a fragment of $\mathbf{L}_{2}$.

\section{§3 Translating First-Order Logic into Propositional Logic}

We will now show that first-order logic is translationally equivalent with propositional logic. In fact, we will show any logic satisfying a few simple properties can be translated into propositional logic.

Definition 3.1 (Monotonic Logic). We will say a logic $\mathbf{L}$ monotonic if for all $\Gamma, \Delta \subseteq \mathcal{L}$ such that $\Gamma \subseteq \Delta$ and for all $\phi \in \mathcal{L}$, if $\Gamma \models_{\mathbf{L}} \phi$, then $\Delta \models_{\mathbf{L}} \phi$.

(Note I am using "monotonic" here in a sense different from the sense of "monotonic" when applied specifically to non-normal modal logics. In what follows, I will only use "monotonic" in the sense of §3.)

Definition 3.2 (Compact Logic). We will say a logic $\mathbf{L}$ is compact if for all $\Gamma \subseteq \mathcal{L}$ and $\phi \in \mathcal{L}, \Gamma \models_{\mathbf{L}} \phi$ only if for some finite $\Gamma_{0} \subseteq \Gamma, \Gamma_{0} \models_{\mathbf{L}} \phi$.

The following result is due to Jeřábek [2012]:

Theorem 3.3 (Jeřábek). Let $\mathbf{L}$ be a compact monotonic logic with at most countably many formulas. Then $\mathrm{L} \rightsquigarrow \mathrm{CPL}$.

Jeřábek provides an explicit construction of the translation and shows that the translation is Turing-equivalent to the consequence relation of the source logic. This is quite general, but the details of the proof are quite involved. What is more, the construction is not guaranteed to produce a translation scheme. This raises the question of whether FOL and CPL are translationally equivalent. We will now show the answer is affirmative. Unlike Jeřábek's constructive proof, our proof will go indirectly via Lindenbaum-Tarski algebras. First, some terminology. A poset $\langle P, \leqslant\rangle$ is a meet-semilattice if every finite subset of $P$ has a greatest lower bound.

Definition 3.4 (Adjunctive Logic). A logic $\mathbf{L}$ is adjunctive if for any $\Gamma \subseteq \mathcal{L}$, if there is a formula $\phi$ such that $[\phi]_{\mathbf{L}}=\bigwedge_{\gamma \in \Gamma}[\gamma]_{\mathbf{L}}$, then $\Gamma \models_{\mathbf{L}} \phi$. We will often write such a $\phi$ as " $\wedge \Gamma$ " given it exists. 
Theorem 3.5. Let $\mathbf{L}_{1}$ and $\mathbf{L}_{2}$ be compact monotonic adjunctive logics. Suppose also that $\mathbb{L}_{1}$ and $\mathbb{L}_{2}$ are meet-semilattices.

(a) $\quad \mathbf{L}_{1} \rightsquigarrow \mathbf{L}_{2}$ iff there is an order-embedding from $\mathbb{L}_{1}$ to $\mathbb{L}_{2}$ that preserves finite meets.

(b) $\quad \mathbf{L}_{1} \leadsto \mathbf{L}_{2}$ iff $\mathbb{L}_{1}$ is order-isomorphic to $\mathbb{L}_{2}$.

(c) $\quad \mathbf{L}_{1} \cong \mathbf{L}_{2}$ iff there is an $f: \mathbb{L}_{1} \cong \mathbb{L}_{2}$ where $\left|[\phi]_{1}\right|=\left|f\left([\phi]_{1}\right)\right|$ for each $\phi \in \mathcal{L}_{1}$.

Proof: The left-to-right directions are straightforward. For the right-to-left directions:

(a) Let $f: \mathcal{L}_{1} / \equiv_{1} \rightarrow \mathcal{L}_{2} / \equiv_{2}$ be an order-embedding that preserves finite meets. For each $[\phi]_{1} \in \mathcal{L}_{1} / \equiv_{1}$, let $f_{[\phi]_{1}}:[\phi]_{1} \rightarrow f\left([\phi]_{1}\right)$ be an arbitrary map. Define $\mathrm{t}(\phi)=f_{[\phi]_{1}}(\phi)$. Since $\mathbf{L}_{1}$ is compact, $\Gamma \models_{1} \phi$ iff for some finite $\Gamma^{\prime} \subseteq \Gamma, \Gamma^{\prime} \models_{1}$ $\phi$. And if $\Gamma^{\prime}$ is finite, then $\Gamma^{\prime} \models_{1} \phi$ iff $\bigwedge \Gamma^{\prime} \models_{1} \phi\left(\bigwedge \Gamma^{\prime}\right.$ exists since $\mathbb{L}_{1}$ is a meet-semilattice). Likewise, $\mathrm{t}[\Gamma] \models_{2} \mathrm{t}(\phi)$ iff $\mathrm{t}\left[\Gamma^{\prime}\right] \models_{2} \mathrm{t}(\phi)$ for some finite $\Gamma^{\prime} \subseteq \Gamma$, and $\mathrm{t}\left[\Gamma^{\prime}\right] \models_{2} \mathrm{t}(\phi)$ iff $\bigwedge \mathrm{t}\left[\Gamma^{\prime}\right] \models_{2} \mathrm{t}(\phi)$. Since $f$ preserves finite meets, $\bigwedge_{\gamma \in \Gamma^{\prime}} f\left([\gamma]_{1}\right)=f\left(\bigwedge_{\gamma \in \Gamma^{\prime}}[\gamma]_{1}\right)=f\left(\left[\bigwedge \Gamma^{\prime}\right]_{1}\right)$. Thus, $\bigwedge \mathrm{t}\left[\Gamma^{\prime}\right] \equiv_{2} \mathrm{t}\left(\bigwedge \Gamma^{\prime}\right)$. So to show that $\mathrm{t}$ is a translation, it suffices to show that for any $\phi, \psi \in \mathcal{L}_{1}, \phi \models_{1} \psi$ iff $\mathrm{t}(\phi) \models_{2} \mathrm{t}(\psi)$. But $\phi \models_{1} \psi$ iff $[\phi]_{1} \leqslant_{1}[\psi]_{1}$, iff $f\left([\phi]_{1}\right) \leqslant_{2} f\left([\psi]_{1}\right)$, iff $\mathrm{t}(\phi) \models_{2} \mathrm{t}(\psi)$. So $\mathrm{t}: \mathbf{L}_{1} \rightsquigarrow \mathbf{L}_{2}$.

(b) Let $f: \mathcal{L}_{1} / \equiv_{1} \rightarrow \mathcal{L}_{2} / \equiv_{2}$ be an order-isomorphism. As before, for each $[\phi]_{1} \in$ $\mathcal{L}_{1} / \equiv_{1}$, let $f_{[\phi]_{1}}:[\phi]_{1} \rightarrow f\left([\phi]_{1}\right)$ be an arbitrary map. Likewise, for each $[\psi]_{2} \in \mathcal{L}_{2} / \equiv_{2}$, let $g_{[\psi]_{2}}:[\psi]_{2} \rightarrow f^{-1}\left([\psi]_{2}\right)$ be arbitrary. Define $\mathrm{t}(\phi)=$ $f_{[\phi]_{1}}(\phi)$ and $\mathbf{s}(\psi)=g_{[\psi]_{2}}(\psi)$. The reasoning above shows that $\mathrm{t}: \mathbf{L}_{1} \rightsquigarrow \mathbf{L}_{2}$ and $\mathrm{s}: \mathbf{L}_{2} \rightsquigarrow \mathbf{L}_{1}$. Now, let $\phi \in \mathcal{L}_{1}$. Then $\phi \equiv_{1} \mathbf{s}(\mathrm{t}(\phi))$ iff $[\phi]_{1}=[\mathrm{s}(\mathrm{t}(\phi))]_{1}=$ $f^{-1}\left([\mathrm{t}(\phi)]_{2}\right)=f^{-1}\left(f\left([\phi]_{1}\right)\right)=[\phi]_{1}$. So $\phi \equiv_{1} \mathrm{~s}(\mathrm{t}(\phi))$ for all $\phi \in \mathcal{L}_{1}$. Likewise, $\psi \equiv \equiv_{1} \mathrm{t}(\mathrm{s}(\psi))$ for all $\psi \in \mathcal{L}_{2}$. Hence, $\mathrm{t}, \mathrm{s}: \mathbf{L}_{1} \leadsto \mathbf{L}_{2}$.

(c) Under these conditions, we can take each $f_{[\phi]_{1}}$ to be bijective, making $\mathrm{t}$ as a whole bijective.

\section{Corollary 3.6. FOL $\cong \mathrm{CPL}$.}

Proof: Immediate since $\mathbb{L}_{\mathrm{FOL}}$ and $\mathbb{L}_{\mathrm{CPL}}$ are countable atomless Boolean algebras and any two countable atomless Boolean algebras are isomorphic.

Note that such an isomorphism is obviously undecidable. One might try to block this result by requiring notational variants to be Turing-equivalent. But this requirement is both too weak and too strong. On the one hand, it is too weak, since monadic first-order logic, which is decidable, would still be deemed to be a notational variant of propositional 
logic. On the other hand, it is too strong, since it seems plausible that some notational variants of a logic can be more computationally efficient than others. We can illustrate this point with a simple example.

Example 3.7. Let $X \subseteq \mathbb{N}$ be a nonrecursive set, and let $\mathcal{L}_{\text {Prop }}(\bowtie)$ be the result of adding countably many binary connectives $\bowtie_{i}$ (where $i \in \mathbb{N}$ ) to $\mathcal{L}_{\text {Prop }}$. We will define the logic CPL ${ }^{\bowtie}$ semantically. The semantics for atomics and the standard boolean connectives is the same as before. The semantics of $\bowtie_{i}$ is as follows: if $i \in X$, then $\phi \bowtie_{i} \psi$ is true on a valuation iff $\phi$ and $\psi$ are true on that valuation; if $i \notin X$, then $\phi \bowtie_{i} \psi$ is true on a valuation iff $\phi$ or $\psi$ is true on that valuation. Finally, $\mathbf{C P L} \mathbf{C}^{\bowtie}=$ $\left\langle\mathcal{L}_{\text {Prop }}(\bowtie), \models_{\bowtie}\right\rangle$, where $\Gamma \models_{\bowtie} \phi$ iff $\phi$ is true on every valuation on which $\Gamma$ is true. Intuitively, $\mathbf{C P L}{ }^{\bowtie}$ is a notational variant of CPL. After all, each $\bowtie_{i}$ is definable in terms of connectives in CPL: CPL $(\bowtie)$ is just CPL with infinitely many connectives expressing conjunction or disjunction. But CPL ${ }^{\bowtie}$ is not decidable, since a decision procedure for $\mathbf{C P L}^{\bowtie}$ would generate a decision procedure for $X$ (just check to see if $\left.p \models_{\bowtie} p \bowtie_{i} q\right)$.

Thus, we cannot avoid Theorem 3.5 by appealing to computability considerations. Something else must explain why FOL and CPL are not merely notational variants.

Corollary 3.6 allows us to define a $t$ and s such that $t, s: F O L ~ \leadsto$ CPL that preserves the boolean connectives exactly:

Proposition 3.8. There are some $\mathrm{t}, \mathrm{s}$ : FOL $\leadsto \mathrm{CPL}$ such that $\mathrm{t}(\neg \phi)=\neg \mathrm{t}(\phi)$ and $\mathrm{t}(\phi \wedge \psi)=\mathrm{t}(\phi) \wedge \mathrm{t}(\psi)$ (and likewise for $\mathrm{s}$ ).

Proof: Let $i$ : FOL $\rightsquigarrow$ CPL be bijective. Define $\mathrm{t}$ and $\mathrm{s}$ as follows:

$$
\begin{array}{rlrl}
\mathrm{t}\left(P^{n}\left(y_{1}, \ldots, y_{n}\right)\right) & =i\left(P^{n}\left(y_{1}, \ldots, y_{n}\right)\right) & \mathrm{s}(p) & =i^{-1}(p) \\
\mathrm{t}(\neg \phi) & =\neg \mathrm{t}(\phi) & \mathrm{s}(\neg \phi) & =\neg \mathrm{s}(\phi) \\
\mathrm{t}(\phi \wedge \psi) & =\mathrm{t}(\phi) \wedge \mathrm{t}(\psi) & \mathrm{s}(\phi \wedge \psi) & =\mathrm{s}(\phi) \wedge \mathrm{s}(\psi) \\
\mathrm{t}(\forall x \phi) & =i\left(\forall x i^{-1}(\mathrm{t}(\phi))\right) . &
\end{array}
$$

It is straightforward to check by induction that $\mathrm{t}(\phi) \equiv_{\mathrm{CPL}} i(\phi)$ for all $\phi \in \mathcal{L}_{\text {Pred }}$ and $\mathbf{s}(\psi) \equiv_{\text {FOL }} i^{-1}(\psi)$ for all $\psi \in \mathcal{L}_{\text {Prop. }}$. Hence, for any $\phi \in \mathcal{L}_{\text {Pred }} \mathbf{s}(\mathrm{t}(\phi)) \equiv_{\text {FOL }}$ $i^{-1}(i(\phi))=\phi$. Likewise, for any $\psi \in \mathcal{L}_{\text {Prop }} \mathrm{t}(\mathrm{s}(\psi)) \equiv_{\mathrm{CPL}} i\left(i^{-1}(\psi)\right)=\psi$. So $\mathrm{t}, \mathrm{s}:$ FOL $\leadsto$ CPL.

It is interesting to note that there is no isomorphism between FOL and CPL with this property. If there were such an $i$, then it would have to map both atomic predicate formulas and quantified formulas to atomic propositional formulas (e.g., if $i(F(x))=\neg \theta$, then $F(x)=i^{-1}(i(F(x)))=i^{-1}(\neg \theta)=\neg i^{-1}(\theta)$, contrary to the fact that $F(x)$ has no negation, \{) . But then $i(\forall x F(x))$ and $i(F(x))$ would need to be logically independent atomic formulas, contrary to the fact that $\forall x F(x) \models_{\text {FOL }} F(x)$, . 


\section{\$4 Compositionality \& Schematicity}

The notion of a translation as defined in Definition 2.2 is fairly minimal. In theory, a translation could be quite gerrymandered and complex. In practice, most translations that have been studied are fairly schematic. Usually one defines a translation by first defining how to translate the atomic formulas, and then settling how to define the translation of complex formulas in terms of their parts via another schema. And indeed, the translations from FOL to CPL described in Theorem 3.5 and in Proposition 3.8 do not have this property. This suggests the thesis that two logics are notational variants just in case they are translationally equivalent via schematic translations. In this section, we will explore different ways of fleshing out this idea.

First, to explicate this idea more precisely, we need to build more structure into the definition of a logic. As it stands, a logic is just a class of formulas together with a consequence relation on those formulas. Nothing in Definition 2.1 demands that the class of formulas a logic is built from must have any underlying compositional structure. Thus, if we want to make use of the notion of schematicity, the definition of a logic must include a specification of its underlying syntactic structure.

Definition 4.1 (Signature). A signature is a pair $\Sigma=\langle A t, 0 p\rangle$ where At and $0 p$ are nonempty classes and $0 \mathrm{p}$ is a class of pairs $\langle\Delta, \gamma\rangle$ where $\Delta$ is a set and $\gamma$ is an ordinal. The $\Sigma$-syntax is the smallest class $\mathcal{L}_{\Sigma}$ such that:

(i) for all $\phi \in \mathrm{At},\langle\phi\rangle \in \mathcal{L}_{\Sigma}$

(ii) for all $\langle\triangle, \gamma\rangle \in$ Op and all $\rho \in \mathcal{L}_{\Sigma}^{\gamma}$ (= the class of $\gamma$-sequences of elements of $\left.\mathcal{L}_{\Sigma}\right),\langle\triangle, \rho\rangle \in \mathcal{L}_{\Sigma}$ (we may write " $\triangle(\rho)$ " in place of " $\langle\triangle, \rho\rangle$ ").

We call the members of $\mathcal{L}_{\Sigma}$ the $\Sigma$-formulas. A $\Sigma$-logic is a pair $\langle\Sigma, \models\rangle$ where $\Sigma$ is a signature and $\left\langle\mathcal{L}_{\Sigma}, \models\right\rangle$ is a logic in the sense of Definition 2.1. A translation from $\mathbf{L}_{1}=\left\langle\Sigma_{1}, \models_{1}\right\rangle$ to $\mathbf{L}_{2}=\left\langle\Sigma_{2}, \models_{2}\right\rangle$ is just a translation from $\left\langle\mathcal{L}_{\left.\Sigma_{1}, \models_{1}\right\rangle}\right\rangle\left\langle\mathcal{L}_{\Sigma_{2}}, \models_{2}\right\rangle$.

A number of authors have claimed that for two logics to be notational variants, there need to exist some compositional translations between them. ${ }^{6}$ To make this precise, we need the following definition:

Definition 4.2 (Schema). Let $\Sigma=\langle\mathrm{At}, 0 \mathrm{p}\rangle$ be a signature, and let $\Pi$ be disjoint from $\mathcal{L}_{\Sigma}$. A $\Sigma$-schema with parameters in $\Pi$ is a $\Sigma(\Pi)$-formula where $\Sigma(\Pi)=\langle$ At $\cup \Pi, 0 p\rangle$. If $\rho \in \mathcal{L}_{\Sigma}^{\gamma}$ and if $\Theta(\pi)$ is a $\Sigma$-schema where $\pi$ is a $\gamma$-sequence listing the parameters in $\Theta$, we may write " $\Theta(\rho)$ " for the $\Sigma$-formula obtained by replacing each $\pi(\beta)$ in $\Theta(\pi)$ with $\rho(\beta)$ for $\beta<\gamma$.

6Epstein [1990, p. 391] uses the term "grammatical" instead of "compositional". Kracht and Wolter [1999, p. 100], Pelletier and Urquhart [2003, p. 269] and Caleiro and Gonçalves [2007, p. 108] build compositionality into the definition of a translation from the start. 
Definition 4.3 (Compositionality). Let $\mathbf{L}_{1}$ and $\mathbf{L}_{2}$ be $\Sigma_{1}$ - and $\Sigma_{2}$-logics respectively. A translation $\mathrm{t}$ : $\mathbf{L}_{1} \rightsquigarrow \mathbf{L}_{2}$ is compositional if for all $\Delta \in \mathrm{p}_{1}$, there is an $\Sigma_{2}$-schema $\Theta^{\triangle}(\pi)$ such that for all $\rho \in \mathcal{L}_{1}^{\gamma}, \mathrm{t}(\triangle(\rho))=\Theta^{\triangle}(\mathrm{t} \circ \rho)$.

The existence of a translation from one logic to another does not in general imply the existence of a compositional translation from the former to the latter. In particular, there is no compositional translation from FOL to CPL, nor one from IPL to CPL. ${ }^{7}$ On the other hand, there is a compositional translation from CPL to FOL and a compositional translation from CPL to IPL. Compositional translations can also be used to distinguish CPL and most normal modal logics:

Proposition 4.4. If $\mathrm{L}$ is a normal modal logic and if $\mathrm{t}: \mathrm{L} \rightsquigarrow \mathrm{CPL}$ is compositional, then $\square \phi \equiv_{\mathbf{L}} \phi \vee \square \perp$.

Proof: Suppose $\Theta^{\square}(\pi)$ is a $\mathcal{L}_{\mathrm{CPL}}$-schema such that $\mathrm{t}(\square \phi)=\Theta^{\square}(\mathrm{t}(\phi))$. Observe that $\Theta^{\square}(\mathrm{t}(\phi)) \equiv \mathrm{CPL}(\mathrm{t}(\phi) \wedge \lambda) \vee(\neg \mathrm{t}(\phi) \wedge \mu)$, where $\lambda$ and $\mu$ are some $\mathcal{L}_{\mathrm{CPL}}$-formulas. Since $\models_{\mathbf{L}} \square T$, we have that $\models_{\mathrm{CPL}} \mathrm{t}(\square T) \equiv_{\mathrm{CPL}}(\mathrm{t}(T) \wedge \lambda) \vee(\neg \mathrm{t}(T) \wedge \mu) \equiv_{\mathrm{CPL}} \lambda$. Hence, $\mathrm{t}(\square \phi) \equiv_{\mathrm{CPL}} \mathrm{t}(\phi) \vee \mu$. Thus, $\mathrm{t}(\phi) \vDash_{\mathrm{CPL}} \mathrm{t}(\square \phi)$, and so $\phi \vDash_{\mathbf{L}} \square \phi$, from which it follows that $\square \phi \equiv_{\mathbf{L}} \phi \vee \square \perp$.

Most translations that have been studied in the literature are compositional. So one might suspect we could simply postulate that two logics are notational variants just in case they are compositionally translationally equivalent. But this would be too restrictive. For instance, a number of modal logicians see the van Benthem characterization theorem as showing that modal logic is just (a notational variant of) the bisimulation-invariant fragment of first-order logic via the standard translation. ${ }^{8}$ But the standard translation of modal logic into first-order logic is not compositional according to Definition 4.3. ${ }^{9}$ In particular, consider the $\square$-clause:

$$
S T_{n}(\square \phi)=\forall x_{n+1}\left(R\left(x_{n}, x_{n+1}\right) \rightarrow S T_{n+1}(\phi)\right) .
$$

Since $S T_{n}(\phi)$ does not occur anywhere as a subformula of $S T_{n}(\square \phi)$ (rather, $S T_{n+1}(\phi)$ does), and since compositional translations are required to have the translations of their constituents as subformulas, $S T_{n}$ is not compositional. In fact, it can be shown that there is no compositional $\mathrm{t}: \mathbf{K} \rightsquigarrow \mathbf{F O L}$, where $\mathbf{K}$ is the minimal normal modal logic. The following is proved in the appendix: ${ }^{10}$

${ }^{7}$ This follows from Theorems 4.7 and 4.8 below. There are also more direct proofs of these claims. For instance, suppose there were a compositional $t:$ FOL $\rightsquigarrow$ CPL. Then where $\Theta$ is the CPL-schema such that $\mathrm{t}(\forall x \phi)=\Theta(\mathrm{t}(\phi))$, we have $\mathrm{t}(\forall x \top)=\Theta(\mathrm{t}(\top)) \equiv_{\text {CPL }} \Theta(\top)$. Hence, $\mathrm{t}(\phi) \models_{\text {CPL }} \mathrm{t}(\phi) \leftrightarrow \top \models$ CPL $\Theta(\mathrm{t}(\phi)) \leftrightarrow$ $\Theta(\top) \models$ CPL $\Theta(t(\phi))=\mathrm{t}(\forall x \phi)$ for any $\phi \in \mathcal{L}_{\text {Pred. }}$. But then $\phi \models$ FOL $\forall x \phi$ for any $\phi \in \mathcal{L}_{\text {Pred }},\{$.

${ }^{8}$ See, e.g., Andréka et al. [1998, p. 1] and Blackburn et al. [2001, p. 70].

${ }^{9}$ Mossakowski et al. Mossakowski et al. [2009, p. 4] make this observation as well, though they do not offer any alternative notion in its place.

${ }^{10}$ The theorem cannot be extended to all normal modal logics, since there is a compositional translation 
Theorem 4.5. Let $\mathbf{L}$ be a normal modal logic. If $\mathrm{t}: \mathrm{L} \rightsquigarrow \mathbf{F O L}$ is compositional, then $\square \phi \equiv_{\mathbf{L}} \square \square \phi$.

Hence, it would be too restrictive to demand that notational variants be compositionally translationally equivalent. Still, arguably there is a sense in which the standard translation is nearly compositional. The problem with the definition of compositionality (Definition 4.3) is that sometimes a translation can only be defined simultaneously with other translations. This is what the standard translation of modal logic into first-order logic illustrates. But intuitively, that should not matter. What is important is not that the translation of a complex formula is strictly a schema of the translation of the parts, but rather that the translation of a complex formula is uniform and fixed solely by its syntactic structure. This motivates a more general notion of compositionality along the following lines: ${ }^{11}$

Definition 4.6 (Schematicity). Let $\mathbf{L}_{1}$ and $\mathbf{L}_{2}$ be $\Sigma_{1}$ - and $\Sigma_{2}$-logics respectively, and let $T$ be a class of translations from $\mathbf{L}_{1}$ to $\mathbf{L}_{2}$. We will say $T$ is compositionally interdependent if for each $\mathrm{t} \in T$ and for each $\triangle \in 0 \mathrm{p}_{1}$, there is an $\Sigma_{2}$-schema $\Theta^{\triangle}(\pi)$ with a $\gamma$-sequence of distinct parameters $\pi$ and there is a $\tau \in T^{\gamma}$ such that for all $\rho \in \mathcal{L}_{1}^{\gamma}, \mathrm{t}(\triangle(\rho))=\Theta^{\triangle}(\tau \cdot \rho)$, where we define $(\tau \cdot \rho)(\beta)=\tau(\beta)(\rho(\beta))$. We will say a translation is schematic if it is a member of a compositionally interdependent set. ${ }^{a}$

\footnotetext{
${ }^{a}$ We could also require schematic translations to translate atomic formulas schematically. Such a constraint seems well-motivated, but it was not included in this definition for purposes of generality, as it was not necessary in the results to follow.
}

If $\mathrm{t}$ is compositional, then it is a member of a compositionally interdependent set, but not vice versa, as the standard translation from $\mathbf{K}$ into FOL shows. So the fact that no compositional translation from FOL to CPL exists does not immediately imply that there is no schematic translation from FOL to CPL. Fortunately, with a little more work, we can achieve this result as well.

Theorem 4.7. There is no schematic $t:$ FOL $\rightsquigarrow$ CPL.

Proof: Suppose there were such a t. Let $\Theta(\pi)$ be a $\Sigma_{\text {CPL }}$-schema with a single parameter $\pi$ and let $\mathrm{t}^{\prime}:$ FOL $\rightsquigarrow$ CPL be such that $\mathrm{t}(\exists x \phi)=\Theta\left(\mathrm{t}^{\prime}(\phi)\right)$ (such a schema must exist if there are such schemas for $\forall x$ and $\neg)$. Then $\models_{\mathrm{CPL}} \mathrm{t}(\top) \equiv_{\mathrm{CPL}} \mathrm{t}(\exists x \top)=$ $\Theta\left(\mathrm{t}^{\prime}(T)\right) \equiv_{\mathrm{CPL}} \Theta(T)$ (since $\left.\mathrm{t}^{\prime}(T) \equiv_{\mathrm{CPL}} \top\right)$. Hence, $\mathrm{t}^{\prime}(\phi) \vDash_{\mathrm{CPL}} \mathrm{t}^{\prime}(\phi) \leftrightarrow \top \models_{\mathrm{CPL}}$ $\Theta\left(\mathrm{t}^{\prime}(\phi)\right) \leftrightarrow \Theta(T) \vDash \mathrm{CPL} \Theta\left(\mathrm{t}^{\prime}(\phi)\right)=\mathrm{t}(\exists x \phi)$. So $\mathrm{t}^{\prime}(\phi) \models_{\mathrm{CPL}} \mathrm{t}(\exists x \phi)$ for all $\phi \in$ $\mathcal{L}_{\text {FOL }}$.

$$
\text { Now, } \Theta\left(\mathrm{t}^{\prime}(\phi)\right), \neg \mathrm{t}^{\prime}(\phi) \models \mathrm{CPL} \Theta(\perp) \text {. Moreover, } \neg \mathrm{t}^{\prime}(\perp) \models \mathrm{CPL} \mathrm{t}^{\prime}(\perp) \leftrightarrow \perp \models \mathrm{CPL}
$$

from S5 to FOL (setting $\mathrm{t}(\square \phi)=\forall x \mathrm{t}(\phi))$. It is unknown whether the result extends to other logics like S4 that validate $\square \phi \leftrightarrow \square \square \phi$.

${ }^{11}$ The definition is inspired by the definition of "recursive" translations from French [2010, p. 16], who attributes the definition to Steven Kuhn. 
$\Theta\left(\mathrm{t}^{\prime}(\perp)\right) \leftrightarrow \Theta(\perp)$. So $\mathrm{t}(\exists x \phi), \neg \mathrm{t}^{\prime}(\phi), \neg \mathrm{t}^{\prime}(\perp) \models \mathrm{CPL} \Theta\left(\mathrm{t}^{\prime}(\perp)\right)$. But $\mathrm{t}^{\prime}(\perp) \models \mathrm{CPL}$ $\Theta\left(\mathrm{t}^{\prime}(\perp)\right)$, so either way, $\mathrm{t}(\exists x \phi), \neg \mathrm{t}^{\prime}(\phi) \vDash \mathrm{CPL} \Theta\left(\mathrm{t}^{\prime}(\perp)\right)=\mathrm{t}(\exists x \perp) \equiv \mathrm{CPL} \mathrm{t}(\perp)$. Hence, $\mathrm{t}(\exists x \phi) \models \mathrm{CPL} \mathrm{t}^{\prime}(\phi) \vee \mathrm{t}(\perp)$. Moreover, the converse holds too, since $\mathrm{t}^{\prime}(\phi) \models \mathrm{CPL}$ $\mathrm{t}(\exists x \phi)$ and $\mathrm{t}(\perp) \models_{\mathrm{CPL}} \mathrm{t}(\exists x \phi)$. So $\mathrm{t}(\exists x \phi) \equiv_{\mathrm{CPL}} \mathrm{t}^{\prime}(\phi) \vee \mathrm{t}(\perp)$ for all $\phi \in \mathcal{L}_{\text {FOL }}$. Now, observe that if s: FOL $\rightsquigarrow \mathbf{C P L}, \mathrm{s}(\phi \wedge \psi) \equiv \mathrm{CPL} \mathrm{s}(\phi) \wedge \mathrm{s}(\psi)$ for any $\phi, \psi \in \mathcal{L}_{\text {FOL }}$. Thus, we have $\mathrm{t}(\exists x \phi \wedge \exists x \neg \phi) \equiv \mathrm{CPL} t(\exists x \phi) \wedge \mathrm{t}(\exists x \neg \phi) \models \mathrm{CPL}\left(\mathrm{t}^{\prime}(\phi) \wedge \mathrm{t}^{\prime}(\neg \phi)\right) \vee$ $\mathrm{t}(\perp) \equiv_{\mathrm{CPL}} \mathrm{t}^{\prime}(\phi \wedge \neg \phi) \vee \mathrm{t}(\perp) \equiv \mathrm{CPL} \mathrm{t}^{\prime}(\perp) \vee \mathrm{t}(\perp) \equiv_{\mathrm{CPL}} \mathrm{t}(\exists x \perp) \equiv_{\mathrm{CPL}} \mathrm{t}(\perp)$, 亿.

We have yet to find a natural example of a pair of $\operatorname{logics} \mathbf{L}_{1}$ and $\mathbf{L}_{2}$ that are schematically intertranslatable but not schematically translationally equivalent. Given Proposition 2.10, one might wonder whether IPL and CPL could witness schematic intertranslatability without schematic translational equivalence. The answer is negative:

Theorem 4.8. There is no schematic $t:$ IPL $\rightsquigarrow$ CPL.

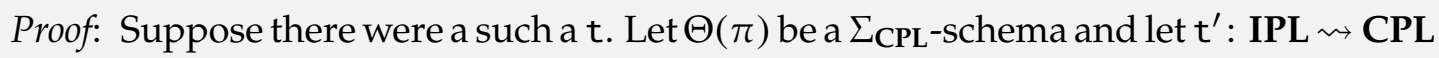
be such that $\mathrm{t}(\neg \phi)=\Theta\left(\mathrm{t}^{\prime}(\phi)\right)$. Then $\models$ CPL $\mathrm{t}(T)=\mathrm{t}(\neg \perp)=\Theta\left(\mathrm{t}^{\prime}(\perp)\right)$. So $\models_{\mathrm{CPL}}$ $\Theta\left(\mathrm{t}^{\prime}(\perp)\right)$. So $\mathrm{t}^{\prime}(\neg \phi) \vDash \mathrm{CPL} \mathrm{t}^{\prime}(\phi \leftrightarrow \perp) \vDash \mathrm{CPL} \mathrm{t}^{\prime}(\phi) \leftrightarrow \mathrm{t}^{\prime}(\perp) \models \mathrm{CPL} \Theta\left(\mathrm{t}^{\prime}(\phi)\right) \leftrightarrow$ $\Theta\left(\mathrm{t}^{\prime}(\perp)\right) \models \mathrm{CPL} \Theta\left(\mathrm{t}^{\prime}(\phi)\right)$. So $\mathrm{t}^{\prime}(\neg \phi) \models \mathrm{CPL} \mathrm{t}(\neg \phi)$.

Since $\models_{\mathrm{CPL}} \mathrm{t}^{\prime}(\perp) \vee \neg \mathrm{t}^{\prime}(\perp)$ and $\models \mathrm{CPL} \Theta\left(\mathrm{t}^{\prime}(\perp)\right)$, we have that $\models_{\mathrm{CPL}} \Theta(T) \vee \Theta(\perp)$. Now, $\mathrm{t}(\perp) \vDash \mathrm{CPL} \mathrm{t}(\neg \phi)$; so $\neg \mathrm{t}(\neg \phi) \models_{\mathrm{CPL}} \neg \mathrm{t}(\perp) \equiv \mathrm{CPL} \neg \mathrm{t}(\neg \top)=\neg \Theta\left(\mathrm{t}^{\prime}(\top)\right) \equiv_{\mathrm{CPL}}$ $\neg \Theta(T) \models_{\mathrm{CPL}} \Theta(\perp) \models_{\mathrm{CPL}} \Theta\left(\mathrm{t}^{\prime}(\neg \phi)\right)\left(\right.$ since $\neg \mathrm{t}(\neg \phi) \models_{\mathrm{CPL}} \neg \mathrm{t}^{\prime}(\neg \phi) \models_{\mathrm{CPL}} \mathrm{t}^{\prime}(\neg \phi) \leftrightarrow$ $\perp)$. Thus, $\neg \mathrm{t}(\neg \phi) \models_{\mathrm{CPL}} \Theta\left(\mathrm{t}^{\prime}(\neg \phi)\right)=\mathrm{t}(\neg \neg \phi)$. Hence, $\models_{\mathrm{CPL}} \mathrm{t}(\neg \phi) \vee \neg \mathrm{t}(\neg \phi) \models_{\mathrm{CPL}}$ $\mathrm{t}(\neg \phi) \vee \mathrm{t}(\neg \neg \phi)$. But $\mathrm{t}(\phi) \vee \mathrm{t}(\psi) \models$ CPL $\mathrm{t}(\phi \vee \psi)$. So $\vDash$ CPL $\mathrm{t}(\neg \phi \vee \neg \neg \phi)$, even though $\not \|_{\text {IPL }} \neg \phi \vee \neg \neg \phi$.

These results suggest that a more adequate precisification of the concept of notational variance can be stated in terms of schematicity: two logics are notational variants just in case they are schematically translationally equivalent.

This is not to say that schematic translational equivalence is the correct precisification of notational variance. Perhaps one will find this particular precisification too restrictive or too general, in which case one might want to explore other notions of notational variance for different purposes. It might turn out that there simply is no unique precisification of this informal concept. Still, schematic translational equivalence at least seems to be an improvement over other notions in the literature in its ability to align more closely with our intuitive judgments.

\section{§5 Conclusion}

Translations are often employed as a way of determining whether or not one logic is a notational variant of a fragment of another. We saw, however, that most attempts to precisify the concept of a notational variant using translations are either too weak or too strong. If, on the one hand, we stipulate that translational equivalence is sufficient for notational 
variance, then we will be forced to say that first-order logic and propositional logic are notational variants. If, on the other hand, we require notational variants to be compositionally intertranslatable, then modal logic will not be a notational variant of a fragment of first-order logic. Fortunately, we saw that we could balance between these two proposals by stipulating that two logics are notational variants just in case they are schematically translationally equivalent. Thus, equating notational variance with schematic translational equivalence seems to be a plausible alternative to the previous accounts of notational variance.

\section{§A Proof of Theorem 4.5}

Let $\Theta(\xi)$ be a first-order schema such that $t(\square \phi)=\Theta(t(\phi))$. Without loss of generality, we may assume $\Theta(\xi)$ is in (roughly) prenex normal form, i.e., that:

$$
\Theta(\mathrm{t}(\phi))=\mathrm{Q}_{1} y_{1} \ldots \mathrm{Q}_{n} y_{n}((\mathrm{t}(\phi) \wedge \lambda) \vee(\neg \mathrm{t}(\phi) \wedge \mu))
$$

where $\lambda$ and $\mu$ are boolean combinations of atomic FOL-formulas and each $Q_{i} \in\{\forall, \exists\}$. Observe that:

$$
\models_{\mathrm{FOL}} \mathrm{t}(\square \top)=\mathrm{Q}_{1} y_{1} \ldots \mathrm{Q}_{n} y_{n}((\mathrm{t}(\top) \wedge \lambda) \vee(\neg \mathrm{t}(\top) \wedge \mu)) \equiv_{\mathrm{FOL}} \mathrm{Q}_{1} y_{1} \ldots \mathrm{Q}_{n} y_{n} \lambda .
$$

So $\models_{\text {FOL }} Q_{1} y_{1} \ldots Q_{n} y_{n} \lambda$.

First, we show $\square \phi \models_{\mathrm{L}} \square \square \phi$. Using the fact that $\models_{\text {FOL }} \mathrm{Q}_{1} y_{1} \ldots \mathrm{Q}_{n} y_{n} \lambda$ :

$$
\begin{aligned}
\mathrm{t}(\square \phi) & \models_{\text {FOL }} \mathrm{t}(\square \phi) \wedge \mathrm{Q}_{1} y_{1} \ldots \mathrm{Q}_{n} y_{n} \lambda \\
& \equiv_{\text {FOL }} \mathrm{Q}_{1} y_{1} \ldots \mathrm{Q}_{n} y_{n}(\mathrm{t}(\square \phi) \wedge \lambda),
\end{aligned}
$$

since $y_{1}, \ldots, y_{n}$ are already bound in $\mathrm{t}(\square \phi)$. So:

$$
\begin{aligned}
\mathrm{t}(\square \phi) & \models_{\text {FOL }} \mathrm{Q}_{1} y_{1} \ldots \mathrm{Q}_{n} y_{n}(\mathrm{t}(\square \phi) \wedge \lambda) \\
& \models_{\text {FOL }} \mathrm{Q}_{1} y_{1} \ldots \mathrm{Q}_{n} y_{n}((\mathrm{t}(\square \phi) \wedge \lambda) \vee(\neg \mathrm{t}(\square \phi) \wedge \mu)) \\
& =\mathrm{t}(\square \square \phi) .
\end{aligned}
$$

Hence, $\mathrm{t}(\square \phi) \models_{\text {FOL }} \mathrm{t}(\square \square \phi)$, and thus, $\square \phi \models_{\mathbf{L}} \square \square \phi$.

Next, we show $\square \square \phi \models_{\mathbf{L}} \square \phi$. Observe that:

$$
\Theta(\mathrm{t}(\phi)) \equiv_{\mathrm{FOL}} \mathrm{Q}_{1} y_{1} \ldots \mathrm{Q}_{n} y_{n}((\mathrm{t}(\phi) \vee \mu) \wedge(\neg \mathrm{t}(\phi) \vee \lambda))
$$

So:

$$
\begin{aligned}
\mathrm{t}(\square \square \phi) & \equiv \text { FOL }_{1} y_{1} \ldots \mathrm{Q}_{n} y_{n}((\mathrm{t}(\square \phi) \vee \mu) \wedge(\neg \mathrm{t}(\square \phi) \vee \lambda)) \\
& \models_{\text {FOL }} \mathrm{Q}_{1} y_{1} \ldots \mathrm{Q}_{n} y_{n}(\mathrm{t}(\square \phi) \vee \mu) \\
& \equiv_{\text {FOL }} \mathrm{t}(\square \phi) \vee \mathrm{Q}_{1} y_{1} \ldots \mathrm{Q}_{n} y_{n} \mu \\
& \equiv_{\text {FOL }} \mathrm{t}(\square \phi) \vee\left(\neg \mathrm{t}(\square \phi) \wedge \mathrm{Q}_{1} y_{1} \ldots \mathrm{Q}_{n} y_{n} \mu\right) \\
& \equiv_{\text {FOL }}\left(\mathrm{t}(\square \phi) \wedge \mathrm{Q}_{1} y_{1} \ldots \mathrm{Q}_{n} y_{n} \lambda\right) \vee\left(\neg \mathrm{t}(\square \phi) \wedge \mathrm{Q}_{1} y_{1} \ldots \mathrm{Q}_{n} y_{n} \mu\right) \\
& \equiv_{\text {FOL }} \mathrm{Q}_{1} y_{1} \ldots \mathrm{Q}_{n} y_{n}(\mathrm{t}(\square \phi) \wedge \lambda) \vee \mathrm{Q}_{1} y_{1} \ldots \mathrm{Q}_{n} y_{n}(\neg \mathrm{t}(\square \phi) \wedge \mu)
\end{aligned}
$$




$$
\begin{aligned}
& \models_{\text {FOL }} \mathrm{Q}_{1} y_{1} \ldots \mathrm{Q}_{n} y_{n}((\mathrm{t}(\square \phi) \wedge \lambda) \vee(\neg \mathrm{t}(\square \phi) \wedge \mu)) \\
& =\mathrm{t}(\square \square \phi) .
\end{aligned}
$$

Thus, in particular, $\mathrm{t}(\square \square \phi) \equiv_{\text {FOL }} \mathrm{t}(\square \phi) \vee \mathrm{Q}_{1} y_{1} \ldots \mathrm{Q}_{n} y_{n} \mu$. Now, note that $\mathrm{t}(\phi \wedge \psi) \equiv_{\text {FOL }}$ $\mathrm{t}(\phi) \wedge \mathrm{t}(\psi)$. Hence, unpacking $\mathrm{t}(\square(\phi \wedge \psi))$ :

$$
\begin{aligned}
\mathrm{t}(\square(\phi \wedge \psi)) & \equiv{ }_{\text {FOL }} \mathrm{Q}_{1} y_{1} \ldots \mathrm{Q}_{n} y_{n}((\mathrm{t}(\phi \wedge \psi) \wedge \lambda) \vee(\neg \mathrm{t}(\phi \wedge \psi) \wedge \mu)) \\
& \equiv_{\text {FOL }} \mathrm{Q}_{1} y_{1} \ldots \mathrm{Q}_{n} y_{n}((\mathrm{t}(\phi \wedge \psi) \wedge \lambda) \vee(\neg(\mathrm{t}(\phi) \wedge \mathrm{t}(\psi)) \wedge \mu)) \\
& \equiv_{\text {FOL }} \mathrm{Q}_{1} y_{1} \ldots \mathrm{Q}_{n} y_{n}((\mathrm{t}(\phi \wedge \psi) \wedge \lambda) \vee((\neg \mathrm{t}(\phi) \vee \neg \mathrm{t}(\psi)) \wedge \mu)) \\
& \equiv_{\text {FOL }} \mathrm{Q}_{1} y_{1} \ldots \mathrm{Q}_{n} y_{n}((\mathrm{t}(\phi \wedge \psi) \wedge \lambda) \vee(\neg \mathrm{t}(\phi) \wedge \mu) \vee(\neg \mathrm{t}(\psi) \wedge \mu))
\end{aligned}
$$

Since $\square(\phi \wedge \psi) \models_{\mathrm{L}} \square \phi$, and since $\mathrm{Q}_{1} y_{1} \ldots \mathrm{Q}_{n} y_{n}(\neg \mathrm{t}(\psi) \wedge \mu) \models_{\text {FOL }} \mathrm{t}(\square(\phi \wedge \psi)$ (given the last equivalence above), that means that $\mathrm{Q}_{1} y_{1} \ldots \mathrm{Q}_{n} y_{n}(\neg \mathrm{t}(\psi) \wedge \mu) \models_{\text {FOL }} \mathrm{t}(\square \phi)$ for any $\phi$ and $\psi$. In particular, $\mathrm{Q}_{1} y_{1} \ldots \mathrm{Q}_{n} y_{n}(\neg \mathrm{t}(\square \phi) \wedge \mu) \equiv_{\text {FOL }} \neg \mathrm{t}(\square \phi) \wedge \mathrm{Q}_{1} y_{1} \ldots \mathrm{Q}_{n} y_{n} \mu \models_{\text {FOL }}$ $\mathrm{t}(\square \phi)$. Hence, $\mathrm{Q}_{1} y_{1} \ldots \mathrm{Q}_{n} y_{n} \mu \models_{\text {FOL }} \mathrm{t}(\square \phi)$. Thus, we have that $\mathrm{t}(\square \square \phi) \equiv_{\text {FOL }} \mathrm{t}(\square \phi)$.

\section{References}

Andréka, Hajnal, Németi, István, and van Benthem, Johan. 1998. "Modal Languages and Bounded Fragments of Predicate Logic." Journal of Philosophical Logic 27:217-274.

Blackburn, Patrick, de Rijke, Maarten, and Venema, Yde. 2001. Modal Logic. Cambridge University Press.

Caleiro, Carlos and Gonçalves, Ricardo. 2007. "Equipollent Logical Systems." Logica Universalis $97-109$.

Epstein, Richard L. 1990. The Semantic Foundations of Logic. Propositional Logics. Kluwer Academic Pub.

French, Rohan. 2010. Translational Embeddings in Modal Logic. Ph.D. thesis.

Gödel, Kurt. 1933. "Zur Intuitionistischen Arithmetik und Zahlentheorie." Ergebnisse eines mathematischen Kolloquiums 4:34-38. Reprinted in Gödel 1986, pp. 286-295.

—. 1986. Collected Works. I: Publications 1929-1936. Oxford University Press.

Jeřábek, Emil. 2012. “The Ubiquity of Conservative Translations." The Review of Symbolic Logic 5:666-678.

Kracht, Marcus and Wolter, Frank. 1999. "Normal monomodal logics can simulate all others." The Journal of Symbolic Logic 64:99-138.

Mossakowski, Till, Diaconescu, Rǎzvan, and Tarlecki, Andrzej. 2009. "What is a logic translation?" Logica Universalis 3:95-124.

Pelletier, Francis Jeffry and Urquhart, Alasdair. 2003. "Synonymous Logics." Journal of Philosophical Logic 32:259-285. 
Straßburger, Lutz. 2007. "What is a Logic, and What is a Proof?" In Logica Universalis, 135-152. Basel: Springer Science \& Business Media.

Thomason, S K. 1974. "Reduction of Tense Logic to Modal Logic. I." The Journal of Symbolic Logic 39:549-551.

-. 1975. "Reduction of tense logic to modal logic II." Theoria 41:154-169.

Wójcicki, Ryszard. 1988. Theory of Logical Calculi. Basic Theory of Consequence Operations. Springer Science \& Business Media. 